\title{
Turbulence Mechanisms of Enhanced Performance Stellarator Plasmas
}

\author{
P. Xanthopoulos $\odot,{ }^{1}$ S. A. Bozhenkov, ${ }^{1}$ M. N. Beurskens, ${ }^{1}$ H. M. Smith, ${ }^{1}$ G. G. Plunk,${ }^{1}$ P. Helander, ${ }^{1}$ C. D. Beidler $\odot,{ }^{1}$ \\ J. A. Alcusón $\odot,{ }^{1}$ A. Alonso, ${ }^{1}$ A. Dinklage, ${ }^{1}$ O. Ford $\odot,{ }^{1}$ G. Fuchert, ${ }^{1}$ J. Geiger, ${ }^{1}$ J. H. E. Proll ${ }^{2}{ }^{2}$ M. J. Pueschel, ${ }^{3}$ \\ Y. Turkin, ${ }^{1}$ F. Warmer $\odot,{ }^{1}$ and the W7-X Team ${ }^{1}$ \\ ${ }^{1}$ Max-Planck-Institut für Plasmaphysik, Wendelsteinstraße 1, 17491 Greifswald, Germany \\ ${ }^{2}$ Eindhoven University of Technology, $5600 \mathrm{MB}$ Eindhoven, The Netherlands \\ ${ }^{3}$ Institute for Fusion Studies, University of Texas at Austin, Austin, Texas 78712, USA
}

(Received 27 February 2020; revised 13 July 2020; accepted 20 July 2020; published 10 August 2020)

\begin{abstract}
We theoretically assess two mechanisms thought to be responsible for the enhanced performance observed in plasma discharges of the Wendelstein 7-X stellarator experiment fueled by pellet injection. The effects of the ambipolar radial electric field and the electron density peaking on the turbulent ion heat transport are separately evaluated using large-scale gyrokinetic simulations. The essential role of the stellarator magnetic geometry is demonstrated, by comparison with a tokamak.
\end{abstract}

DOI: 10.1103/PhysRevLett.125.075001

Introduction.-Stellarator reactors are attractive contenders for the production of magnetic fusion energy, thanks to operation advantages over their tokamak siblings, such as quiescent behavior at large plasma beta, a soft density limit, and the absence of current-driven instabilities or disruptions [1,2]. Furthermore, optimized stellaratorslike the Wendelstein 7-X (W7-X) [3], the world's largest fusion facility of the stellarator type-employ sophisticated magnetic field designs to restrict neoclassical transport losses, thus raising the importance of turbulent processes for the explanation of energy and particle transport [4].

The recent campaign OP1.2 of the W7-X stellarator experiment has revealed a broad class of improved confinement discharges, for which the heat transport across some flux surfaces is largely neoclassical. Such discharges involve transient phases characterized by enhanced performance, following shortly after the injection of cryogenic hydrogen pellets into an electron cyclotron resonance (ECR)-heated plasma [5]. The energy confinement during these phases is notably better than that in gas fueled discharges at similar density and heating power, and exceeds the empirical stellarator scaling ISS04 [6], leading also to a high triple product [7].

These remarkable findings suggest that turbulence mitigation might underlie pellet enhanced performance (PEP) scenarios in optimized stellarators. To assess this possibility, we separately investigate two mechanisms for the

Published by the American Physical Society under the terms of the Creative Commons Attribution 4.0 International license. Further distribution of this work must maintain attribution to the author(s) and the published article's title, journal citation, and DOI. Open access publication funded by the Max Planck Society. reduction of the ion-scale turbulent transport in the W7-X fusion device driven by the ion temperature and density gradients (for aspects of electron-scale turbulence driven by the electron temperature gradient, the reader is referred to [8]), described as follows: (i) Turbulence reduction via ambipolar electric field.-In stellarator geometry, the radial electric field $E_{r}$, derived numerically as the root of the ambipolarity condition using the code DKES [9], induces a poloidal rotation on the magnetic surface, which, if strong enough, can dislodge the density fluctuations from their natural position into regions where the curvature is relatively weak. As a direct implication of this feature, the nonlinear ion heat transport is reduced. We note that this mechanism is distinct from the $E \times B$ shearing observed at the tokamak edge [10,11], which relies on the radial derivative of the field, $d E_{r} / d r$. Although the impact of shearing on stellarator turbulence still evades explicit numerical evaluation, it is thought to play a leading role for the formation of electron internal transport barriers in various stellarator experiments [12-14]. In our scenario, however, the electric field attains its extreme value at the radial location of interest, and therefore shearing should be much less significant. (ii) Turbulence reduction via electron density peaking. - The discharge phase shortly after the pellet injection is accompanied by the formation of a strong localized electron density gradient, coexisting with the ion temperature gradient. This combination leads to a transition for the turbulence, from the usual ion temperature gradient (ITG) type, without or with a subcritical density gradient, to the so-called ion-driven trapped electron mode (ITEM) type. The ITEM $[15,16]$ feeds on available ion free energy and propagates in the ion diamagnetic direction. More importantly, it encompasses the dynamics of electrons being trapped due to the variation of the magnetic field. Electron trapping occurs also in tokamak 
configurations, however the entire trapped population is found on the low field side of the device, and the linear instability is generally exacerbated as the density gradient increases [17]. In the W7-X stellarator, thanks to a specific magnetic field optimization that separates the bulk of trapped electrons from the strong curvature regions $[18,19]$, the density gradient is instead responsible for the amelioration of the instability [20].

Experimental data.-The W7-X program 20181016.037 is employed in the standard configuration. We focus on two phases of the discharge, see Fig. 1: (i) The ECR phase $t=(4-5) \mathrm{s}$, during which the effect of the pellets has vanished long after their injection into the plasma; this phase is essentially equivalent to the initial ECR-heated part of the discharge, prior to pellet injection. (ii) The PEP phase $t=(1.65-1.75) \mathrm{s}$, taking place shortly after the pellet injections. During this phase, the experimental heat transport is reduced almost to neoclassical levels, as a power balance analysis indicates [21], and the diamagnetic energy $W_{\text {dia }}$ reaches its maximum value following a sharp increase. In Fig. 1 (bottom) we summarize normalized gradients and the radial electric field, serving as input for the gyrokinetic simulations for the two discharge phases. Focusing on the radial location where the electric field reaches its most negative value, corresponding to the ion root solution of the ambipolarity relation [22], we notice that the PEP phase is characterized by both larger normalized electron density gradient $a / L_{n_{e}}=\left(-a / n_{e}\right) d n_{e} / d r$ and ion temperature gradient $a / L_{T_{i}}$ compared to the ECR phase ( $a$ is the minor radius of the device).

Simulation setup.-The numerical simulations are performed using the nonlinear gyrokinetic Eulerian solver GENE $[23,24]$ in two different models of operation: (i) The surface model takes into account an entire magnetic flux surface, while the radial direction is treated locally. This model has been already exploited for the investigation of stellarator-specific turbulence features [25], and here it has been extended to include the radial electric field. Introducing the neoclassical electrostatic potential in the Lagrange equation [26], the term (in normalized units) $\left(a^{2} / \imath r T_{e}\right) E_{r} \partial_{\alpha} \tilde{f}$ appears in the gyrokinetic Vlasov equation, where $l$ is the rotational transform, $\alpha$ is the coordinate parametrizing the field line, and $\tilde{f}$ is the perturbed ion distribution function. In addition, $T_{e}$ is the electron temperature in kiloelectron volts and $E_{r}$ is the ambipolar radial electric field in kilovolts per meter. The electrons are assumed to be Boltzmann distributed (adiabatic). This treatment is justified, since the radial electric field induces a rotation with frequency comparable to the ITG drift frequency, which, in turn, is only weakly modified by the kinetic electron dynamics. (ii) The local model is a widespread numerical code, based on the flux-tube concept [27], according to which, a single field line on a magnetic surface is followed, and a slender computational box is constructed around it. Contrary to the surface model, the local model is
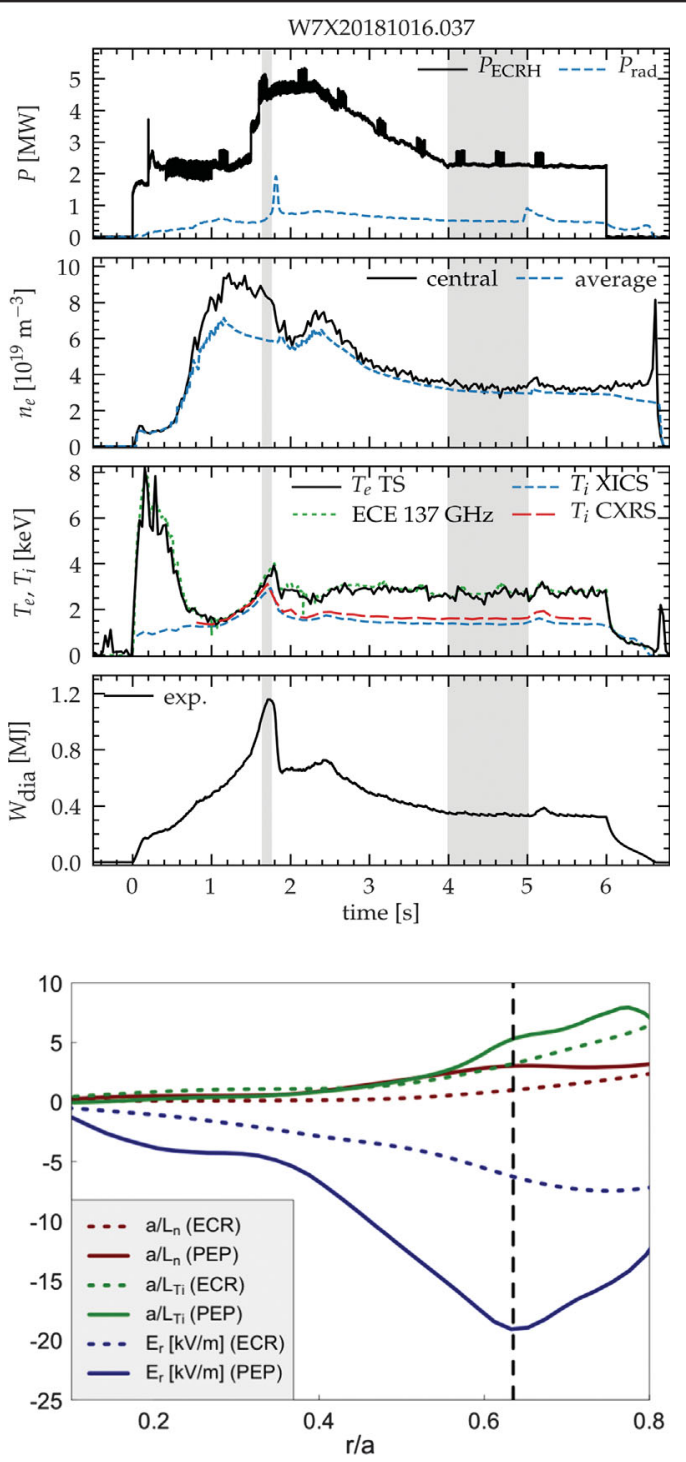

FIG. 1. Time traces of the W7-X program 20181016.037 for heating power $(P)$, electron density $\left(n_{e}\right)$, electron and ion temperatures $\left(T_{e}, T_{i}\right)$, and diamagnetic energy $\left(W_{\mathrm{dia}}\right)$. In the bottom panel, the normalized gradients and radial electric field for the ECR and PEP phases, derived from DKES calculations, are shown. The black line marks the radial location where the simulations are conducted.

furnished with the full gyrokinetic electron response. It is thus suitable for the analysis of the density peaking effect, which relies on the dynamics of trapped electrons. We note in passing that the local model has been shown in stellarator simulations with adiabatic electrons [28] to accurately reproduce the transport level derived from the surface model along the same magnetic field line, if $\rho_{i} / a$ is small enough. The numerical simulations are performed, for both GENE models, at the radial position $r / a=0.63$. For the local model, the magnetic field line $\alpha=0$ is selected, overlapping with the strongest curvature region at the outer midplane of the bean-shaped $(\phi=0)$ cross section. 


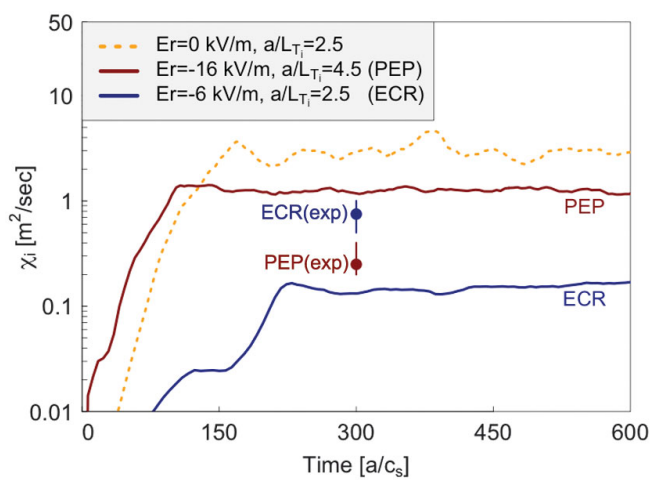

FIG. 2. Comparison of simulated ion heat diffusivities for the ECR and PEP phases of the W7-X pellet discharge, and an artificial case $\left(E_{r}=0\right)$, isolating the role of the radial electric field $\left(a / L_{n_{e}}=0\right)$. The Mach number for the ECR and PEP phases is $0.8 \%$ and $2 \%$, respectively. The discretization of the five-dimensional computational box reads $\left(n_{x}, n_{y}, n_{z}, n_{v_{\|}}, n_{\mu}\right)=$ $64 \times 64 \times 102 \times 30 \times 8$. The size of the computational domain in the radial and binormal directions is $L_{x}=L_{y}=125$ in units of $\rho_{s}=c_{s} / a$, with $c_{s}$ the ion sound speed, and for the parallel direction is $L_{z}=2 \pi a$. The extent for the parallel velocity is $-3 \leq v_{\|} \leq 3$ in units of ion thermal velocity, and for the magnetic moment $0 \leq \mu \leq 9 T_{i} / B_{0}$. Here, $B_{0}=2 \Phi_{0} / a^{2}$, where $\Phi_{0}$ is the toroidal magnetic flux within the last closed flux surface. Also shown are the experimental diffusivities [ECR(exp), PEP(exp)] for the corresponding phases of the discharge (the error bars reflect uncertainties in the measured profiles).

According to the measured profiles, we set $T_{e}=T_{i}$, as expected in the periphery of the plasma, where ions and electrons are well coupled through energy exchange.

Simulation results. -We begin by showing, in a general context, how turbulence can be modified by the unsheared radial electric field $E_{r}$. To isolate this effect, the density gradient has been set to zero, therefore the turbulence is ITG driven. In Fig. 2 we compare ion heat diffusivities from the simulation for the ECR phase (using the parameters from Fig. 1) versus a simulation with the same parameters, but artificially removing the electric field $\left(E_{r}=0\right)$. We find that the ECR simulation, i.e., including the electric field, has notably smaller ion heat diffusivity. The result can be explained by the displacement of the density fluctuations on the magnetic surface. This feature is depicted in Fig. 3, where we compare the spatial distribution for the fluctuations. While in the simulation without electric field the strongest turbulence fluctuations are almost centered around the magnetic field line where the curvature is most unfavorable $(\alpha=0)$, in the ECR simulation the stripe is shifted away from this curvature region. The poloidal rotation velocity relative to the ion sound speed scales with the parameter $\rho_{c}^{*}=\rho_{i} / L_{c}$, where $L_{c}$ is a characteristic length, approximated by the curvature variation on the magnetic surface. In the W7-X stellarator $L_{c} \ll a$ holds, but in a tokamak $L_{c} \approx a$ [28], implying that only stellarators significantly benefit from this kind of stabilization.

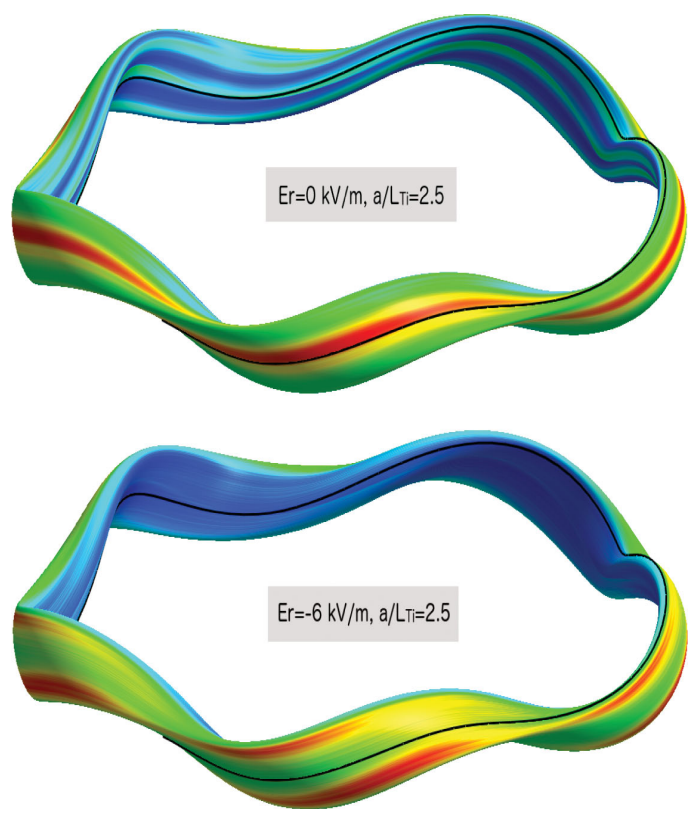

FIG. 3. Time-averaged density fluctuations on the magnetic surface of the W7-X stellarator from the simulations corresponding to Fig. 2 (rescaled with respect to their individual maximum value to facilitate inspection). The magnetic field line $a=0$ is shown in black.

Returning to the numerical estimation of the electric field effect related to our scenario, in Fig. 2 we display ion heat diffusivities from the simulations from the ECR and the PEP phases, together with corresponding experimental values. These simulations falsely predict that the ion heat transport should be stronger in the PEP phase compared to the ECR phase, which means that the electric field effect cannot compensate for the increase of the ion temperature gradient in the PEP phase. This finding suggests that the effect of the density peaking on turbulence should be critical for the experimental validation. In Fig. 4 we compare turbulence features derived from the simulations for the ECR and PEP phases of the discharge. Specifically, we show ion heat diffusivities, demonstrating a significant reduction of the transport level in the PEP phase as compared to the ECR phase. A key observation is the turbulence transition from ITG type (ECR phase) to ITEM type (PEP phase), as illustrated by the different timeaveraged diffusivities along the magnetic field line (inset). The structure for the ECR simulation is characterized by a central portion, "ballooning" around the middle of the magnetic field line, typical of ITG turbulence. After the transition to the ITEM type, however, this portion appears suppressed, and regions of the line responding to the trapped electrons become more pronounced, although still remaining at low amplitudes. Looking at the linear behavior of the instabilities, the ITEM has both smaller growth rate and scale than the ITG mode, a combination which implies significantly lower turbulence based on mixing length 

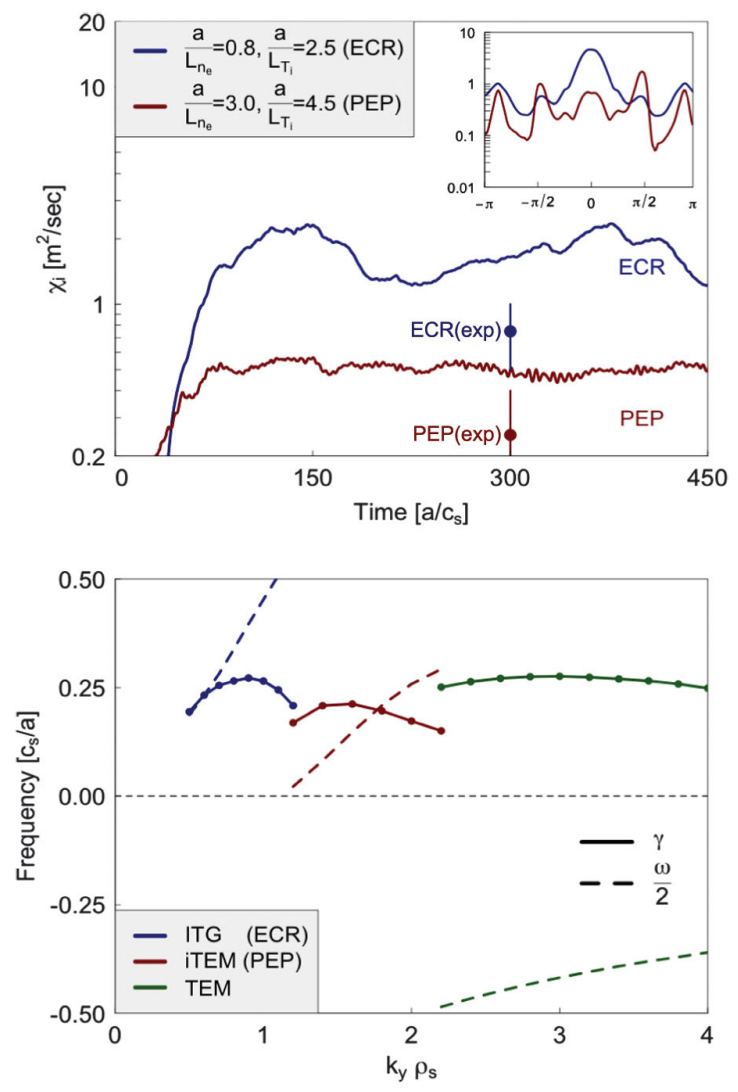

FIG. 4. Comparison of simulated ion heat diffusivities for the ECR and PEP phases of the W7-X pellet discharge, isolating the role of the density peaking $\left(E_{r}=0\right)$. The time-averaged diffusivities for the two phases along the magnetic field line are found in the inset. Also shown are the experimental diffusivities [ECR (exp), PEP(exp)] for the corresponding phases (the error bars reflect uncertainties in the measured profiles). In the bottom panel, the real frequency $(\omega)$ and growth rate $(\gamma)$ spectra for various linear modes, related to the experimental conditions, are displayed. The ITG mode characterizes the ECR phase, while the ITEM the PEP phase. Both modes propagate in the ion diamagnetic direction $(\omega>0)$. The TEM, on the other hand, driven by the density gradient alone, propagates in the electron diamagnetic direction.

arguments. For reference, the trapped electron mode (TEM), driven only by the electron density gradient, is also shown. Similar to the ITG mode, the ITEM propagates in the ion diamagnetic direction, and its real frequency is very small as a result of the fine balance between the TEM and ITG modes. The simulated ion heat diffusivities appear larger than the experimental ones, for each phase of the discharge. The main reason for this discrepancy, we argue, is the suppression of the electric field in these simulations, which is expected to reduce the diffusivity levels, as shown before. Other reasons are the absence of electromagnetic and finite $\rho_{i} / a$ effects, which are both known to cause turbulence reduction $[25,29,30]$. For this particular case, however, due to the relatively small values of the $\beta$
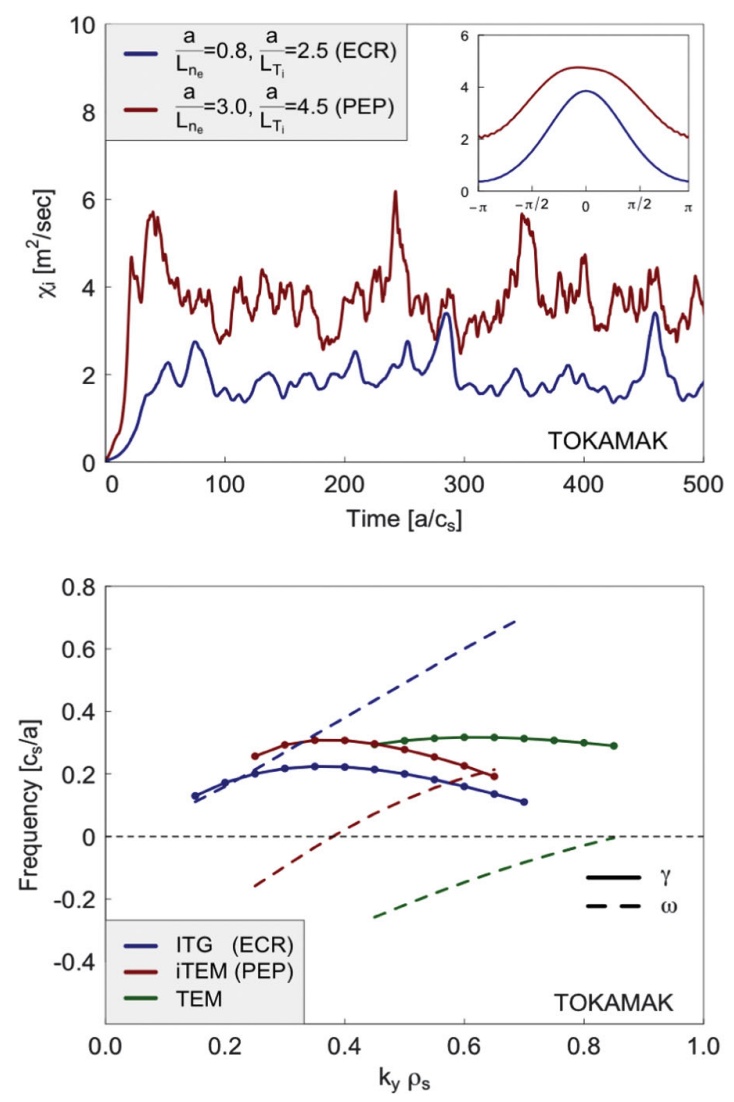

FIG. 5. Comparison of simulated ion heat diffusivities for a tokamak configuration, applying parameters similar to the two phases of the W7-X pellet discharge. In the bottom panel, the real frequency $(\omega)$ and growth rate $(\gamma)$ spectra for the linear modes are shown (see Fig. 4 for details).

parameter $(<1 \%)$ and $\rho_{i} / a\left(\sim 10^{-3}\right)$, these effects should play a minor role.

The density peaking is a markedly different mechanism from those reported in various PEP tokamak experiments, involving either a reversed magnetic shear, due to a large bootstrap current, or high collisionality in medium-scale devices [31-36]. To see why, we employ an axisymmetric configuration with aspect ratio similar to that of $\mathrm{W} 7-\mathrm{X}$ and a standard value for the magnetic shear, $\hat{s}=0.76$ [37]. Using this setup, we impose density gradients resembling the ECR and PEP phases of the W7-X discharge. The resulting ion heat diffusivities are shown in Fig. 5, where the PEP-type simulation predicts stronger transport compared to the ECR-type simulation. This finding is reflected in the time-averaged diffusivities along the magnetic field line (inset), where the structure is much broader due to the destabilizing effect of trapped electrons. For the same reason, the growth rate for the ITEM appears much larger in the tokamak, compared to that for the ITG mode, while the scales for the two modes are similar.

Summary.-Using gyrokinetic simulations to analyze a typical pellet discharge of the W7-X stellarator, we have demonstrated the critical role of the density peaking and the 
secondary role of the ambipolar radial electric field for the explanation of the PEP. While the impact of the electric field alone on turbulence failed to capture the experimental trends, our results nevertheless imply that ion turbulence could be significantly mitigated through a sufficiently strong radial electric field, as exemplified by the "optimum confinement" discharges of the previous stellarator Wendelstein 7-AS [38]. The substantial turbulence reduction due to the density peaking is understood through the transition between two different types of turbulence, namely from ITG to ITEM, the latter being characterized by much lower ion heat transport in an appropriately optimized stellarator device. Despite the transient nature of the turbulence reduction, such PEP regimes could pave the way for the steady-state operation of a fusion reactor, assuming that the density peaking can be sustained. In this respect, scenarios with neutral beam injection heating or a continuous pellet injector will be employed in next experimental campaigns.

We thank F. Jenko, A. Navarro, T. Görler, F. Merz, and D. Told. The simulations were performed on the Cobra and Marconi HPC clusters. This work has been carried out within the framework of the EUROfusion Consortium and has received funding from the Euratom research and training programme 2014-2018 and 2019-2020 under Grant Agreement No. 633053.

The views and opinions expressed herein do not necessarily reflect those of the European Commission.

[1] A. Weller, K. Y. Watanabe, S. Sakakibara, A. Dinklage, H. Funaba, J. Geiger, J. H. Harris, S. Ohdachi, R. Preuss, Y. Suzuki, A. Werner, H. Yamada, M. C. Zarnstorff (the W7-X Team, and the LHD Experimental Group), Nucl. Fusion 49, 065016 (2009).

[2] P. Helander, Rep. Prog. Phys. 77, 087001 (2014).

[3] T. Klinger et al., Fusion Eng. Des. 88, 461 (2013).

[4] A. Dinklage, C. Beidler, P. Helander, G. Fuchert, H. Maassberg, K. Rahbarnia, T. Pedersen, T. Turkin, R. Wolf et al., Nat. Phys. 14, 855 (2018).

[5] J. Baldzuhn, H. Damm, C. D. Beidler, K. McCarthy, N. Panadero, C. Biedermann, S. A. Bozhenkov, K. J. Brunner, G. Fuchert, Y. Kazakov et al., Plasma Phys. Controlled Fusion 61, 095012 (2019).

[6] H. Yamada, J. H. Harris, A. Dinklage, E. Ascasibar, F. Sano, S. Okamura, J. Talmadge, U. Stroth, A. Kus, S. Murakami, M. Yokoyama, C. D. Beidler, V. Tribaldos, K. Y. Watanabe, and Y. Suzuki, Nucl. Fusion 45, 1684 (2005).

[7] T. S. Pedersen, R. König, M. Krychowiak, M. Jakubowski, J. Baldzuhn, S. Bozhenkov, G. Fuchert, A. Langenberg, H. Niemann, D. Zhang et al., Plasma Phys. Controlled Fusion 61, 014035 (2018).

[8] G. Plunk, P. Xanthopoulos, G. M. Weir, S. A. Bozhenkov, A. Dinklage, G. Fuchert, J. Geiger, M. Hirsch, U. Hoefel, M. Jakubowski, A. Langenberg, N. Pablant, E. Pasch,
T. Stange, D. Zhang, and (the W7-X Team), Phys. Rev. Lett. 122, 035002 (2019).

[9] S. P. Hirshman, K. C. Shaing, W. I. van Rij, C. O. Beasley, and E. C. Crume, Phys. Fluids 29, 2951 (1986).

[10] K. Ida, Plasma Phys. Controlled Fusion 40, 1429 (1998).

[11] T. S. Hahm, D. H. Na, J. W. Lee, J. W. Park, Y. S. Na, W. H. Kim, S. S. Ko, P. H. Diamond, H. Jhang, and Y. Jeon, Nucl. Fusion 53, 093005 (2013).

[12] T. Estrada, L. Krupnik, N. Dreval, A. Melnikov, S. M. Khrebtov, C. Hidalgo, B. van Milligen, F. Castejón, E. Ascasíbar, L. Eliseev, A. Chmyga, A. D. Komarov, A. S. Kozachok, and V. Tereshin, Plasma Phys. Controlled Fusion 46, 277 (2004).

[13] J. Lore, W. Guttenfelder, A. Briesemeister, T. Anderson, F. S. B. Anderson, C. B. Deng, K. Likin, D. A. Spong, J. N. Talmadge, and K. Zhai, Phys. Plasmas 17, 056101 (2010).

[14] N. Kenmochi, T. Minami, C. Takahashi, S. Mochizuki, K. Nishioka, S. Kobayashi, K. Nagasaki, Y. Nakamura, H. Okada, S. Kado, S. Yamamoto, S. Ohshima, S. Konoshima, G. M. Weir, Y. Otani, and T. Mizuuchi, Plasma Phys. Controlled Fusion 59, 055013 (2017).

[15] P. Helander, J. Proll, and G. Plunk, Phys. Plasmas 20, 122505 (2013).

[16] G. G. Plunk, J. W. Connor, and P. Helander, J. Plasma Phys. 83, 715830404 (2017).

[17] F. Romanelli and S. Briguglio, Phys. Fluids B 2, 754 (1990).

[18] P. Helander, T. Bird, F. Jenko, R. Kleiber, G. G. Plunk, J. H. E. Proll, J. Riemann, and P. Xanthopoulos, Nucl. Fusion 55, 053030 (2015).

[19] J. H. E. Proll, P. Xanthopoulos, and P. Helander, Phys. Plasmas 20, 122506 (2013).

[20] J. Alcusón, P. Xanthopoulos, G. G. Plunk, P. Helander, F. Wilms, Y. Turkin, A. von Stechow, and O. Grulke, Plasma Phys. Controlled Fusion 62, 035005 (2020).

[21] S. A. Bozhenkov, Y. Kazakov, O. P. Ford, M. N. A. Beurskens, J. Alcusón, J. A. Alonso, J. Baldzhun, C. Brandt, K. J. Brunner, H. Damm et al., Nucl. Fusion 60, 066011 (2020).

[22] D. E. Hastings, W. A. Houlberg, and K. C. Shaing, Nucl. Fusion 25, 445 (1985).

[23] F. Jenko, W. Dorland, M. Kotschenreuther, and B. N. Rogers, Phys. Plasmas 7, 1904 (2000).

[24] T. Görler, X. Lapillonne, S. Brunner, T. Dannert, F. Jenko, S. K. Aghdam, P. Marcus, B. F. McMillan, F. Merz, O. Sauter, D. Told, and L. Villard, Phys. Plasmas 18, 056103 (2011).

[25] P. Xanthopoulos, H. E. Mynick, P. Helander, Y. Turkin, G. G. Plunk, F. Jenko, T. Görler, D. Told, T. Bird, and J. H. E. Proll, Phys. Rev. Lett. 113, 155001 (2014).

[26] A. J. Brizard and T. S. Hahm, Rev. Mod. Phys. 79, 421 (2007).

[27] M. A. Beer, S. C. Cowley, and G. W. Hammett, Phys. Plasmas 2, 2687 (1995).

[28] P. Xanthopoulos, G. G. Plunk, A. Zocco, and P. Helander, Phys. Rev. X 6, 021033 (2016).

[29] M. J. Pueschel and F. Jenko, Phys. Plasmas 17, 062307 (2010).

[30] G. G. Whelan, M. J. Pueschel, P. W. Terry, J. Citrin, I. McKinney, W. Guttenfelder, and H. Doerk, Phys. Plasmas 26, 082302 (2019). 
[31] P. Smeulders et al., Nucl. Fusion 35, 225 (1995).

[32] L. R. Baylor, T. C. Jernigan, S. K. Combs, W. A. Houlberg, M. Murakami, P. Gohil, K. H. Burrell, C. M. Greenfield, R. J. Groebner, C. L. Hsieh, R. J. La Haye, P. B. Parks, G. M. Staebler, The DIII-D Team, G. L. Schmidt, D. R. Ernst, and E. J. Synakowski, Phys. Plasmas 7, 1878 (2000).

[33] O. Gruber et al., Plasma Phys. Controlled Fusion 30, 1611 (1988).
[34] M. Greenwald et al., Phys. Rev. Lett. 53, 352 (1984).

[35] M. Hugon, B. P. van Milligen, P. Smeulders et al., Nucl. Fusion 32, 33 (1992).

[36] F. Romanelli, C. Bourdelle, and W. Dorland, Phys. Plasmas 11, 3845 (2004).

[37] A. M. Dimits et al., Phys. Plasmas 7, 969 (2000).

[38] M. Hirsch et al., Plasma Phys. Controlled Fusion 50, 053001 (2008). 